\title{
Vécu et contraintes lors de l'application d'une nou- velle approche pédagogique: Une étude de cas québécoise en comptabilité.
}

\section{DANIEL COULOMBE, LOUISE GUILBERT, NICOLE LACOMBE}

Université Laval

\section{ROBERT MATHIEU \& HÉLENE RACINE}

Wilfrid Laurier University \& Université Laval

\section{RÉSUMÉ}

Cet article présente l'expérience vécue au Département des sciences comptables de l'Université Laval lors de la réforme des cours du baccalauréat. Cette réforme s'explique par les changements importants qui bouleversent la profession comptable et par l'écart grandissant entre les besoins des employeurs et les compétences et les attitudes professionnelles des finissants en comptabilité. En plus de présenter le contexte dans lequel s'est effectué la réforme, l'article fait état des obstacles rencontrés tout au long des modifications apportées au contenu des cours et, surtout, de l'implantation d'une nouvelle approche pédagogique.

Le nouveau programme est centré sur une approche collaborative par problèmes où la participation de l'étudiant est fortement sollicitée. Un tel changement ne s'est pas fait sans rencontrer de nombreux obstacles. Par exemple, l'approche choisie a créé un niveau d'incertitude élevé chez les professeurs et a exigé, de leur part, d'allouer une partie importante de leur

Les auteurs désirent remercier les participants au Séminaire Caron Bélanger Ernst \&

Young de l'Université Laval pour leurs commentaires. 
allouée au développement de matériel didactique. La perception des étudiants, obtenue à l'aide d'entrevues de groupe, était que les cours ne les préparaient pas adéquatement et que parfois, ils étaient inutiles. Nous proposons certains éléments de solution qui peuvent réduire l'impact des problèmes rencontrés lors de l'implantation d'une nouvelle approche pédagogique qui bouleverse certaines conceptions fortement ancrées.

\section{ABSTRACT}

This paper presents the experience of faculty members of the accounting department at Université Laval in designing and implementing a new curriculum. These changes have resulted from the new requirements of the profession as well as the growing gap between employers' expectations and skills and professional attitudes of graduating accounting students. In addition to presenting the context of these changes, the paper discusses some of the difficulties faced during the implementation of the new curriculum and, more importantly, those faced when introducing a new teaching approach.

The new curriculum is focused on a collaborative approach whereby student participation is solicited. However, several difficulties have been met during the implementation. For instance, faculty members face a higher level of uncertainty in the classroom and must devote more time in developing teaching material. Students' perceptions, obtained through focus groups, were that the new program did not prepare them properly and that some courses were not useful. Throughout the paper, we offer some strategies that can be used to minimize the impact of the difficulties faced when implementing a new teaching approach that can overturn previously firmly held beliefs about appropriate pedagogical methods.

\section{INTRODUCTION}

Le monde des affaires a énormément évolué au cours des dernières décennies. L'expert-comptable doit davantage agir en tant qu'utilisateur de l'information, de gestionnaire, de concepteur ou d'évaluateur de systèmes d'information. La tâche traditionnelle du comptable, soit la préparation et la vérification de données comptables, occupe une place de moins en 
moins importante. Le rapport de recherche du Groupe de travail pancanadien sur la vision de la profession comptable de l'Institut canadien des comptables agréés (ICCA, 1996) considère que la mondialisation des marchés, l'importance accrue accordée au capital intellectuel ainsi que l'étendue des connaissances et compétences nécessaires à l'exercice de la profession sont en partie responsables de ces changements. De plus, selon le rapport de l'International Federation of Accountants (1995), l'intégration des technologies de l'information doit être une priorité pour les professionnels comptables. Tous ces facteurs ont amené le Groupe de travail pancanadien sur la vision de la profession de l'ICCA à conclure «que la profession canadienne de comptable agréé se trouvait à la croisée des chemins et qu'elle devait examiner d'urgence ce qu'est le comptable agréé, ce qu'il fait» (1996, p. 1).

Malgré les changements importants qui bouleversent la profession comptable, l'enseignement de la comptabilité n'a pas évolué aussi rapidement au cours de la même période. Pour s'en convaincre, il suffit de comparer le syllabus d'un cours d'introduction à la comptabilité avec celui d'un cours similaire dispensé il y a dix ou vingt ans pour y constater le peu de changement. L'écart grandissant entre les attentes des employeurs et les habiletés des finissants rendrait ces derniers de plus en plus démunis face aux défis de la profession (Patten \& Williams, 1990). En fait, l'incapacité des universités à adapter leurs programmes de comptabilité aux nouvelles réalités du marché du travail a été soulevée par divers groupes intéressés à la formation des futurs professionnels comptables. Les critiques ont incité l'American Accounting Association $(A A A)^{\prime}$ à former, en 1984, un groupe de travail (le Comité Bedford) ayant comme mandat d'étudier les caractéristiques de la profession ainsi que son expansion et l'état actuel de la formation en sciences comptables. ̇̀ la suite du rapport déposé en 1986 par ce comité, l'American Accounting Association a formé un groupe de travail permanent, l'Accounting Education Change Commission, devant promouvoir, encourager et financer les réformes en enseignement des sciences comptables.

La prise de conscience qu'a suscité les recommandations des différents comités a amené plusieurs universités américaines à entamer des réformes de leurs programmes en sciences comptables. Malheureusement, 
peu de changements ont été observés au Canada. En fait, il n'y a eu que deux universités qui ont rapidement réagi aux recommandations des organismes comptables américains, soit l'Université Laval et l'University of Waterloo. Considérant les similitudes importantes existant entre la profession comptable des deux pays, il est surprenant de noter le peu d'impact que les comités de recherche américains ont eu sur l'enseignement de la comptabilité au Canada. ${ }^{2}$ L'objectif de cet article est de présenter l'expérience du Département des sciences comptables de l'Université Laval dans ses efforts à réformer les cours au baccalauréat tant au niveau de l'approche pédagogique adoptée, des obstacles rencontrés, que des réussites et améliorations possibles. Par cet article, nous désirons, d'une part, partager notre vécu et exposer certaines difficultés qui peuvent attendre tout département désirant innover en pédagogie et, d'autre part, proposer des éléments de solutions.

Avant de décrire les efforts du Département des sciences comptables de l'Université Laval et afin de mieux comprendre le contexte global de la réforme, l'article présente d'abord l'évolution de la réflexion au sujet de la formation en sciences comptables. Ensuite, une description des particularités du programme de premier cycle de ce département cerne davantage le contexte singulier de cette entité de formation. La réforme des cours est, par la suite, présentée en traitant de la structure du nouveau programme, de l'approche pédagogique et des activités d'apprentissage. Par la suite, l'article fait état des divers obstacles rencontrés ainsi que des réussites tout au long du processus, soulignant d'autant plus les efforts d'implantation du Département, et conclut par des pistes d'améliorations possibles lors de l'implantation d'une nouvelle approche.

\section{L'ÉVOLUTION DE LA RÉFLEXION AU SUJET DE LA FORMATION EN SCIENCES COMPTABLES}

\section{Les premières réflexions}

Les réflexions portant sur les modifications à apporter à l'enseignement des sciences comptables remontent à plus de vingt ans. Traditionnellement, l'enseignement des sciences comptables était magistral et axé sur des contenus déclaratifs et techniques. Les programmes tentaient de «couvrir» 
l'ensemble des concepts et des règles techniques propres à la profession. Peu d'efforts étaient consacrés au développement du jugement et des habiletés et attitudes qui y sont associées. Devant l'étendue des changements affectant tant la profession comptable que le monde des affaires en général, l'approche pédagogique traditionnelle est devenue rapidement inadéquate. D'ailleurs, le défi en formation est davantage de rendre l'étudiant autonome et de développer certaines compétences qui lui permettront de faire face aux nouvelles exigences de sa profession.

Tel que mentionné précédemment, ce n'est qu'en 1984 que l'American Accounting Association a formalisé les discussions en créant un comité, appelé le Comité Bedford d'après le nom de son président, ayant comme mandat d'étudier les caractéristiques de la profession ainsi que son expansion et l'état actuel de la formation en sciences comptables. Le comité a conclu, en 1986, que les programmes en sciences comptables ne préparaient pas adéquatement les étudiants à poursuivre une carrière d'experts-comptables. Les travaux de ce comité font état de 28 recommandations spécifiques, reproduites en annexe, touchant deux objectifs généraux: (a) l'enseignement de la comptabilité doit être élargi pour qu'elle soit vue comme une fonction d'information et de communication visant à appuyer la prise de décision de nature économique et (b) les cours doivent mettre l'emphase sur la capacité des étudiants d'apprendre à apprendre afin de les rendre davantage autonomes et prêts à l'apprentissage de toute une vie.

Les modifications proposées par le comité Bedford sont fondamentales puisque la plupart des programmes d'enseignement des sciences comptables en Amérique du Nord mettent l'accent sur la préparation des états financiers et l'apprentissage de normes techniques. Les étudiants ne sont pas appelés à résoudre des problèmes complexes ou non structurés. ${ }^{3}$ Le changement de cap proposé par le Comité Bedford exige donc, entre autres, une redéfinition des conceptions de l'enseignement et de l'apprentissage ainsi qu'une approche pédagogique différente de la part des professeurs.

En réponse aux recommandations du comité Bedford, les directeurs associés des huit grands cabinets d'experts-comptables de l'époque ont formé un groupe de travail. Les membres de ce groupe ont exprimé leur 
vision des compétences et des connaissances que devraient posséder les futurs experts-comptables pour réussir une carrière en comptabilité dans un document intitulé le White Paper (Arthur Andersen \& Co. et al., 1989). En plus de mettre l'accent sur des connaissances spécifiques à la comptabilité ainsi que des connaissances générales, les membres de ce groupe de travail estiment que les étudiants doivent également développer des habiletés intellectuelles, interpersonnelles et de communication. Ils mentionnent également que l'approche d'enseignement favorisée devrait tenir compte de la composante affective de l'apprentissage des étudiants avec l'intention, entre autres, de favoriser l'émergence de certaines attitudes liées au professionnalisme.

Afin de poursuivre la réflexion enclenchée et d'inciter les universitaires à modifier leur enseignement de la comptabilité, l'American Accounting Association a fondé, en 1989, l'Accounting Education Change Commission (AECC) ayant reçu le mandat de promouvoir, d'encourager et de financer les réformes en enseignement des sciences comptables. La grande partie du financement disponible à cet organisme dans l'accomplissement de son mandat provenait de contributions monétaires des huit grands cabinets comptables de l'époque. En effet, à la suite de la publication du White Paper, les grands cabinets comptables se sont impliqués dans les réformes de cours entreprises par les universités américaines en injectant 4 millions de dollars dans la formation en comptabilité. En plus de cet investissement initial, certaines fondations parrainées par des cabinets d'expertscomptables ont financé des réformes spécifiques comme ce fût le cas pour The Coopers \& Lybrand Foundation qui a parrainé The University of Southern California Year 2000 Curriculum Project. ${ }^{4}$

\section{Les travaux de l'Accounting Education Change Commission}

La première prise de position de l'AECC en 1990 entérine la plupart des recommandations du rapport Bedford et du White Paper. D'après l'AECC, les programmes en sciences comptables devraient favoriser le développement de l'autonomie d'apprentissage chez les étudiants. Afin d'atteindre cet objectif, les universités devraient mettre l'accent sur trois éléments essentiels : les attitudes professionnelles, les connaissances et 
les compétences. Premièrement, par attitudes professionnelles, l'AECC vise les attitudes que devrait posséder un professionnel oeuvrant dans le domaine des sciences comptables soit: une sensibilité à la déontologie, une disposition à l'apprentissage permanent, l'empathie, la motivation, la persévérance, le leadership, le dynamisme, la créativité ${ }^{5}$ et la sensibilité à son rôle social. Dans leurs recommandations à cet effet, il est souligné que les programmes universitaires en sciences comptables devraient faire en sorte d'attirer des candidats possédant ces attitudes ou ayant une prédisposition vis-à-vis celles-ci.

Deuxièmement, toujours d'après cette prise de position, les connaissances nécessaires à l'exercice de la profession englobent autant les connaissances comptables qu'une bonne compréhension du domaine des affaires. L'étudiant doit également avoir de bonnes connaissances générales lui permettant de mieux comprendre les liens entre le milieu des affaires et la société. Pour y parvenir, l'AECC propose une structure de programme composée de quatre niveaux allant du contenu général à un contenu plus spécialisé. Dans un premier niveau, la formation serait plus générale et introduirait le développement d'habiletés de communication et d'habiletés intellectuelles, telles que l'analyse critique et la pensée logique. Le second niveau permettrait aux étudiants d'avoir une bonne compréhension de l'environnement des entreprises et de leur fonctionnement interne. Le troisième niveau mettrait l'accent sur une formation générale en comptabilité axée sur l'identification, la mesure, la communication et l'utilisation de l'information comptable. Finalement, le dernier niveau comprendrait une formation spécialisée en comptabilité. D'après le comité Bedford, cette formation plus spécialisée devrait se faire au deuxième cycle et en formation continue.

Pour ce qui est des compétences, l'AECC définit trois grandes catégories qui devraient être développées chez l'étudiant en sciences comptables soit, les compétences de communication, les compétences intellectuelles et interpersonnelles. Le développement des compétences reliées à la communication orale et écrite devrait permettre à l'étudiant d'argumenter adroitement ses opinions en plus d'être capable d'organiser un rapport utilisant diverses sources d'information ou divers médias. En fait, le développement de ces compétences devrait aussi permettre au futur 
expert-comptable d'entrer en communication réelle avec ses collègues et ses clients en faisant preuve d'écoute empathique et d'ouverture aux divers points de vue, aux diverses valeurs et structures organisationnelles.

Les compétences intellectuelles sont définies comme étant la résolution de problèmes non structurés, le processus d'analyse de problèmes et la capacité d'identifier les forces et les faiblesses d'une situation et d'en prédire les effets. L'AECC y inclut également l'exercice du jugement professionnel ainsi que la gestion adéquate du temps alloué aux différentes tâches. Les compétences interpersonnelles sont définies comme étant la capacité de travailler en équipe, la capacité de convaincre les autres et de collaborer avec les autres, le leadership, la gestion de conflits et une ouverture d'esprit face à la diversité. D'après l'AECC et le groupe de travail composé de directeurs associés des grands cabinets comptables, ces compétences sont nécessaires pour œuvrer à l'intérieur d'une organisation et pour développer des contacts avec les clients.

L'AECC croit que cet ensemble d'attitudes, de connaissances et de compétences intégré dans les nouveaux programmes devrait favoriser la préparation des étudiants, non pas à être des professionnels comptables, mais plutôt à devenir des professionnels comptables à la fin de leurs études (Williams, 1993). Dans cette optique, le développement de la capacité d'apprendre à apprendre ${ }^{6}$ prend tout son sens et favorise l'apprentissage de toute une vie.

À la suite des constats de ces différents comités, plusieurs universités américaines ont décidé de modifier en profondeur leurs programmes en sciences comptables. La plupart des universités qui ont initié ces changements ont été fortement financées par l'AECC ou par des organismes comptables privés. Dans certains cas, le financement de ces nouveaux programmes était octroyé à la condition que les universités bénéficiaires de ces fonds rendraient disponible le matériel pédagogique ainsi développé. Par conséquent, il y a eu une collégialité parmi les universités américaines qui a favorisé le développement accéléré de nouveau matériel pédagogique et l'émergence de nouvelles approches d'enseignement. Ces modifications aux programmes comptables ont fait l'objet de diverses présentations lors des congrès de l'American Accounting Association. La présence de plusieurs conférences sur la 
pédagogie universitaire représentait un changement important puisque celle-ci était peu présente dans le passé. De plus, la proportion d'articles traitant de pédagogie dans les publications de l'American Accounting Association ou exposant les modifications apportées aux programmes d'enseignement de la comptabilité par certaines universités était devenue plus importante.

\section{PARTICULARITÉS DU PROGRAMME DE PREMIER CYCLE EN SCIENCES COMPTABLES DE L'UNIVERSITÉ LAVAL}

En général, le développement des programmes de comptabilité au Québec a été effectué principalement par des comptables agréés. ${ }^{7}$ Ce fût le cas, entre autres, du programme en sciences comptables de l'Université Laval. Par conséquent, le programme a toujours été fortement axé sur la préparation des étudiants aux examens de cet ordre professionnel, tout en considérant les exigences propres à l'examen de l'Ordre des comptables en management accrédités (CMA) et à celui de l'Ordre des comptables généraux licenciés (CGA). Le programme, comme la plupart des autres programmes offerts au Québec, se voulait un programme très compartimenté avec un effort d'intégration visible lors des cours de bachotage offerts après le baccalauréat en vue des examens d'admission aux corporations comptables. Les cours étaient orientés vers la préparation et la vérification des états financiers tandis que l'aspect gestion et l'intégration des connaissances étaient très peu présents. En fait, l'emphase était mise sur l'acquisition de connaissances et de «recettes» pouvant servir aux étudiants lors des examens professionnels.

Au début des années 90, plusieurs facteurs ont incité les professeurs du Département des sciences comptables de l'Université Laval à modifier le programme de comptabilité. Premièrement, nous avons constaté qu'il y avait une faible proportion de candidats inscrits dans les cours de première année qui désiraient poursuivre une carrière en comptabilité. Par conséquent, des cours de base axés sur la préparation de l'information comptable semblaient moins pertinents pour la majorité des étudiants inscrits à ces cours. De plus, la tendance des dernières années démontrait que de moins en moins de bons candidats étaient 
attirés vers la profession comptable. En outre, selon Sundem (1994), «les étudiants qui s'inscrivent en comptabilité sont de plus en plus attirés par le côté structuré et certain de la tenue de livre» (p. 45).

Deuxièmement, la quantité de normes comptables que les étudiants devraient maîtriser ne cessait d'augmenter. Ainsi, il devenait de plus en plus illusoire de tenter de tout «couvrir» dans les cours de baccalauréat. Il devenait plus important de rendre les étudiants autonomes face à leur apprentissage que d'augmenter le nombre de cours. Troisièmement, les finissants en sciences comptables démontraient des faiblesses en ce qui concerne l'intégration de la matière, la compréhension du cadre conceptuel de la profession et le jugement critique. En fait, ils tentaient davantage de mémoriser la matière que de la comprendre. Finalement, conjointement à ces facteurs, la réflexion américaine sur l'enseignement de la comptabilité a déclenché un débat parmi les membres du Département des sciences comptables concernant l'approche pédagogique à utiliser pour le baccalauréat.

Au cours des années qui ont précédé la réforme en profondeur des cours de comptabilité, le Département des sciences comptables de l'Université Laval n'est pas demeuré inactif sur le plan pédagogique. Ainsi, plusieurs innovations ont été introduites au fil des années. Par exemple, la taille des cours de première année en comptabilité a été augmentée de 60 à 90 étudiants afin de dégager des fonds servant à des laboratoires en petits groupes axés sur la résolution de problèmes. ${ }^{8}$ Malheureusement, dû au nombre important de laboratoires nécessaires afin d'offrir le service à tous les étudiants, le système est devenu trop lourd pour être conservé. D'autres innovations ont été tentées par la suite. Par exemple, certains cours de trois heures ont été scindés en deux blocs d'une heure et demie. Dans un premier bloc, la théorie était discutée en classe et des cas étaient travaillés dans le deuxième bloc.

Bien que ces innovations aient eu du mérite, la majorité des cours avaient toujours une approche traditionnelle où les étudiants étaient passifs et où peu d'emphase était mise sur la pensée critique ou créative. De plus, le manque d'intégration de la matière et le peu d'effort accordé au développement des compétences et attitudes telles que défines par l'AECC créaient un écart de plus en plus important entre la qualité des 
finissants en sciences comptables et les attentes des employeurs. L'étudiant était formé en tant que préparateur et vérificateur d'états financiers alors qu'en pratique, il devait davantage agir en tant que gestionnaire ou conseiller en gestion. Devant cet état de fait, les membres du Département ont décidé d'imiter certaines universités américaines et de réformer en profondeur le cheminement en sciences comptables.

\section{LA RÉFORME DES COURS EN SCIENCES COMPTABLES À L'UNIVERSITÉ LAVAL}

À la suite de nombreuses discussions et réflexions, le Département des sciences comptables a décidé de modifier radicalement ses cours de premier cycle en adoptant une nouvelle approche pédagogique basée sur une philosophie d'enseignement davantage axée sur l'apprenant. Cette nouvelle approche pédagogique a nécessité un changement de cap tant au niveau du rôle du professeur que de celui de l'étudiant. En fait, le programme est passé d'une approche traditionnelle ou l'étudiant était passif à une approche axée sur l'autonomie des étudiants tout en favorisant le développement du jugement professionnel ainsi que les habiletés et les attitudes qui y sont rattachées. Le programme vise également à développer chez l'étudiant des connaissances théoriques solides et des habiletés intellectuelles qui sont transférables d'un contexte à un autre. Le programme n'est pas centré sur l'acquisition de multiples règles techniques mais plutôt sur une compréhension globale favorisant l'intégration des connaissances.

\section{La structure du nouveau programme en sciences comptables}

Contrairement à plusieurs universités américaines, la totalité du programme de premier cycle en sciences comptables de l'Université Laval a été réformé. ${ }^{9} \mathrm{La}$ structure adoptée est conforme à la première prise de position de l'AECC soit une structure favorisant l'apprentissage général au début du programme pour se diriger graduellement vers des connaissances plus pointues. La structure de contenu disciplinaire utilisée pour la première année du baccalauréat est celle développée par K. Pincus de 1'University of Southern California. Cette structure met davantage l'accent sur l'utilisation de l'information comptable déjà sous 
forme d'états financiers plutôt que sur leur préparation. Les cours de première année sont structurés en fonction des besoins des groupes d'utilisateurs les plus importants soit les propriétaires, les créanciers, les gestionnaires et le gouvernement. Puisque seulement près de $15 \%$ des étudiants de première année en administration se dirigeront par la suite en comptabilité, la nouvelle structure du contenu introduite dans ces cours est davantage pertinente à l'ensemble de la clientèle étudiante.

Le Département offre quatre cours de comptabilité au cours de la deuxième année (comptabilité de gestion, fiscalité, contrôle et comptabilité financière). Puisque les étudiants en administration choisissant une spécialisation autre que la comptabilité doivent nécessairement prendre deux de ces quatre cours, ces derniers présentent également une préoccupation d'utilisateurs de l'information comptable. Par contre, en plus de l'aspect utilisation, une emphase plus prononcée sur l'aspect technique est introduite. Contrairement à l'approche traditionnelle qui mettait l'emphase sur l'acquisition de règles fiscales, comptables ou autres, les cours de deuxième année s'intègrent dans un contexte de gestion qui est propre aux entreprises. Pour ces cours, le Département des sciences comptables a choisi de ne pas adapter du matériel pédagogique déjà développé par une autre université. Il s'est plutôt lancé dans le développement de matériel didactique inédit convenant davantage à ses besoins.

Les cours de dernière année du baccalauréat visent davantage l'acquisition de connaissances spécialisées puisqu'ils ne s'adressent qu'aux étudiants en sciences comptables. Comme pour ceux de deuxième année, les cours ont été entièrement reformés. Tout en préservant l'approche gestion et conseil de gestion qui caractérise les cours du nouveau cheminement en sciences comptables, la matière discutée dans ces cours est davantage pointue, orientée vers les divers champs de la profession. Par exemple, les cours de comptabilité financière et de vérification accordent une plus grande place à la préparation et à la vérification d'états financiers bien que l'aspect utilisation soit toujours présente. Encore une fois, le Département a dû développer du matériel inédit.

Tel que mentionné précédemment, etant donné la quantité croissante de normes comptables, de vérification et de fiscalité ainsi que la 
complexité et la variété des systèmes d'information et des instruments financiers qui inondent le marché des affaires, il devient illusoire de tenter de tout «couvrir» dans les cours du baccalauréat. À l'opposé de l'idée de couvrir du contenu en classe, la nouvelle approche utilisée, qui donne une plus grande place à l'apprenant, adhère plutôt à la notion de «recherche d'informations» (banque de données, Internet, recherches, rapports, etc.) et de «construction de connaissances». Ainsi, les cours du nouveau programme mettent davantage l'accent sur la construction et la mise à jour constante de savoirs pertinents à la résolution de problèmes diversifiés et imprévisibles. Afin d'atteindre ces objectifs, les cours mettent 1'emphase sur des activités qui permettent aux étudiants d'organiser leurs connaissances, de les intégrer tout en favorisant une solide compréhension conceptuelle qui leur permettra de transférer ces connaissances dans de nouveaux contextes.

\section{L'approche pédagogique adoptée et les activités d'apprentissage}

L'approche pédagogique adoptée se caractérise par certains principes directeurs tels que «apprendre en faisant, entraîner plutôt qu'enseigner et développer un dialogue réciproque de réflexion dans l'action entre l'entraîneur et l'étudiant» (Schön, 1987, p. 303). Les cours sont construits afin de rendre l'étudiant plus autonome et responsable face à son apprentissage. Alors que l'étudiant se contentait d'être passif dans l'approche pédagogique traditionnelle, plutôt centrée sur le professeur, celle récemment adoptée se centre le plus possible sur l'étudiant selon une approche par problèmes (Guilbert et Ouellet, 1997). Celui-ci est appelé à être actif lors des cours puisque les activités d'apprentissage le forcent à travailler en équipe et à présenter ses solutions et sa démarche de résolution à l'ensemble de la classe. En fait, l'étudiant doit verbaliser sa pensée afin d'en évaluer les forces et les faiblesses. En procédant ainsi, le professeur incite l'étudiant à structurer sa pensée et à s'évaluer afin d'ajuster son apprentissage. Dans le but d'atteindre ces objectifs, le professeur se doit de créer un climat à l'intérieur duquel l'étudiant se sent en confiance et participe activement. Ces activités sont très similaires à celles rencontrées sur le marché du travail, tant par les thèmes traités que par les interactions entre les membres d'une équipe. 
L'approche pédagogique choisie a nécessité plusieurs changements tant au niveau du rôle du professeur que des activités d'apprentissage faites en classe. Ainsi, au lieu d'enseigner de façon magistrale tout au long du cours, le professeur devient plus un animateur ou un entraîneur qui doit susciter le questionnement, la réflexion et la créativité chez l'étudiant. Il ne doit pas chercher à tout prévoir et à tout planifier. Au contraire, pour bien bâtir sur les acquis de l'étudiant, il se doit de l'inciter à verbaliser sa pensée pour s'en servir comme point de départ. Le professeur doit donc faire face à un niveau d'incertitude beaucoup plus grand car il peut être appelé a prendre des chemins qui lui sont moins familiers. La planification de chaque séance s'élabore autour de thèmes et d'activités prévues. Par contre, le déroulement du cours et les microsujets travaillés sont appelés à varier au gré des discussions. En fait, puisque le nouveau programme place les étudiants devant des problèmes complexes caractérisés par la contingence, le professeur doit démontrer des compétences à gérer l'incertitude et l'ambiguité et devient ainsi un modèle pour les étudiants. Cette approche s'inspire du socioconstructivisme où les étudiants construisent leur savoir en s'appuyant sur leurs connaissances antérieures, le recueil d'information, leur logique, leur savoir empirique et surtout, la confrontation des idées entre les pairs et les données de base (états financiers, étude de cas, etc.) et ceci, sous la supervision du professeur qui sert de guide (Guilbert \& Ouellet, 1997).

Un élément crucial au déroulement des cours du nouveau programme est le développement d'ateliers d'apprentissage qui favorisent l'émergence des diverses compétences visées. Dans l'ancien programme, la résolution de problèmes simples à solution unique était souvent la seule activité faite en classe, au tableau. La réforme a amené le professeur à utiliser différentes activités. Ainsi, on retrouve dans les cours de première année des débats, des jeux de rôle, des simulations, 1'écoute active de vidéos, des présentations faites par l'étudiant et évaluées par ses pairs et des travaux nécessitant la collaboration d'entreprises du marché des affaires. Ces activités cherchent, entre autres, à développer une certaine autonomie chez l'étudiant et des compétences de communication écrites et orales. ${ }^{10}$ Les compétences de communication sont d'autant plus 
importantes que les praticiens qui réussissent davantage sont, d'après Friedlan (1995), d'excellents communicateurs.

De telles activités sont toujours présentes dans les cours de deuxième et de troisième années. En outre, ces cours mettent davantage l'accent sur des exercices multiples d'application technique afin de favoriser le développement de certains automatismes. La résolution de problèmes complexes ou non structurés conserve toutefois une place importante. Tout comme dans les cours de première année, les étudiants sont appelés à travailler régulièrement en équipe et à présenter divers travaux. Dans certains cours, les présentations des étudiants sont évaluées soit par une équipe de professeurs ou par le professeur responsable du cours et des praticiens tels que des associés de cabinets comptables.

L'interaction constante entre le professeur et l'étudiant, l'emphase mise sur l'autonomie de l'étudiant et le développement de compétences intellectuelles, de communication et interpersonnelles, sont conformes aux recommandations des divers comités tant canadiens qu'américains qui se sont penchés sur l'enseignement de la comptabilité au cours des quinze dernières années. En ne cherchant plus à enseigner une multitude de règles techniques et de «recettes», le nouveau programme incite davantage l'étudiant à développer une compréhension théorique solide au sujet des contenus disciplinaires, mais aussi des compétences et des attitudes professionnelles variées qui pourraient lui permettre de s'adapter à de nouveaux contextes d'application. En fait, comme l'ont mentionné Anderson et Taylor (1994), «la capacité de résoudre des problèmes et le jugement professionnel sont des qualités essentielles à la réussite d'un comptable».

En résumé, la nouvelle philosophie d'enseignement qui caractérise la réforme de la formation en sciences comptables à l'Université Laval a nécessité un changement majeur au niveau de l'approche pédagogique, des stratégies d'enseignement, des activités d'apprentissage et du matériel didactique de soutien. Le tableau 1 fait ressortir plusieurs aspects de ce changement en présentant une comparaison des caractéristiques de l'approche traditionnelle et de la nouvelle approche d'enseignement/ apprentissage adoptée par le Département des sciences comptables. 
Comparison de l'approche traditionnelle avec la nouvelle approche de formation en comptabilité adoptée par le Département des sciences comptables de l'Université Laval

\begin{tabular}{|c|c|}
\hline Approche Traditionnelle & Nouvelle Approche \\
\hline $\begin{array}{l}\text { - Emphase importante mise sur la technique comptable dans } \\
\text { les cours; }\end{array}$ & $\begin{array}{l}\text { - Emphase sur une formation plus large, plus générale, axée sur } \\
\text { les organisations et la gestion; }\end{array}$ \\
\hline $\begin{array}{l}\text { - Les cours d'introduction mettent l'emphase sur un contenu de } \\
\text { préparateurs de l'information financière (processus comptable, } \\
\text { entrées de journal, raports et états financiers); }\end{array}$ & $\begin{array}{l}\text { - Les cours d'introduction mettent l'emphase sur les utilisateurs } \\
\text { de l'information et le rôle de la comptabilité dans la société et } \\
\text { les organisations; emphase sur l'utilisation de l'information } \\
\text { comptable pour la prise de décisions économiques; }\end{array}$ \\
\hline $\begin{array}{l}\text { - Peu d'intégration effectuée entre les cours et dans les cours; les } \\
\text { sujets sont traités de manière compartimentée; }\end{array}$ & $\begin{array}{l}\text { Intégration sensible dans les cours et entre les cours des diverses } \\
\text { disciplines comptables: fiscalité, comptabilité financière, } \\
\text { comptabilité de gestion et vérification; }\end{array}$ \\
\hline $\begin{array}{l}\text { - Utilisation de problèmes bien définis comme outil } \\
\text { d'enseignement et d'apprentissage; problèmes exigeant une } \\
\text { performance très technique et menant à une seule bonne réponse; }\end{array}$ & $\begin{array}{l}\text { Emphase sur la résolution de problèmes nonstructurés tels que } \\
\text { les études de cas; }\end{array}$ \\
\hline $\begin{array}{l}\text { Toute l'informatios nécessaire à la résolution d'un problème est } \\
\text { fournie à l'étudiant; }\end{array}$ & $\begin{array}{l}\text { - Accroissement des situations où l'information doit être évaluée } \\
\text { quant à sa crédibilité, sa pertinence et sa suffisance; identification } \\
\text { par l'étudiant de l'information manquante; }\end{array}$ \\
\hline $\begin{array}{l}\text { - Emphase sur la détermination de LA bonne réponse à un } \\
\text { problème donné; }\end{array}$ & $\begin{array}{l}\text { - Emphase sur l'identification et l'évaluation de diverses solutions } \\
\text { acceptables et adaptées au contexte du problème; }\end{array}$ \\
\hline $\begin{array}{l}\text { - Emphase sur l'enseignement de règles et la «transmission» de } \\
\text { connaissances; }\end{array}$ & $\begin{array}{l}\text { - Emphase sur le processus de réflexion et de résolution de } \\
\text { problèmes ainsi que la construction de connaissances; }\end{array}$ \\
\hline $\begin{array}{l}\text { - Très peu ou pas de préoccupations concernant le processus } \\
\text { d'apprentissage et sa relation avec l'enseignement; }\end{array}$ & $\begin{array}{l}\text { - Accroissement des préoccupations concernant le processus } \\
\text { d'apprentissage et la nécessité de développer l'apprentissage à } \\
\text { apprendre; }\end{array}$ \\
\hline
\end{tabular}


- Enseignement très orienté vers l'examen final uniforme;

- Emphase sur des habiletés intellectuelles simples telles que l'acquisition et le rappel, la compréhension et l'application;

- Très peu d'emphase sur les habiletés de communication et les habiletés interpersonnelles;

- Une seule stratégie d'enseignement: le cours magistral;

- Centration sur le professeur, son enseignement et le contenu à «(couvrir»);

- Les étudiants sont des récepteurs passifs de connaissances;

- Très peu d'utilisation de la technologie informatisée à l'extérieur des cours de système d'information;

- Environnement compétitif;

- Apprentissage de connaissances «par coeun» et évaluation basée sur la reproduction de ces connaissances.
- Reconnaissance d'objectifs plus larges que celui de l'examen final uniforme; objectifs plutôt orientés vers la formation de meilleurs professionnels comptables

- Emphase sur des habiletés intellectuelles de haut niveau telles que l'analyse, la synthèse, l'évaluation, la pensée critique et créative et la résolution de problèmes;

- Accroissement dans la formation des occasions de présentations orales, de productions écrites et de travaux d'équipe exigeant éventuellement la résolution de conflits cognitifs;

- Stratégies pédagogiques diversifiées incluant l'approche par problèmes

- Centration sur l'étudiant et son apprentissage; enseignement favorisant le développement de compétences et d'attitudes professionnelles;

- Les étudiants sont des participants actifs dans la construction de leurs connaissances; enseignement favorisant le développement de l'autonomie intellectuelle;

- Accroissement de l'utilisation de l'ordinateur à travers les programmes de premier et de deuxième cycles;

- Environnement et atmosphère de coopération;

- Apprentissage par la réflexion, l'expression et l'échange de ces réflexions; évaluation plutôt basée sur la production d'idées et la résolution de problèmes. 


\section{L'EXPÉRIENCE VÉCUE PAR LE DÉPARTEMENT DES SCIENCES COMPTABLES}

L'objectif de cette section est de présenter l'expérience vécue par le Département des sciences comptables lors de l'implantation de la réforme. Dans la première sous-section, les principaux obstacles rencontrés sont discutés. Dans la sous-section suivante, les réussites du nouveau programme et des stratégies visant à minimiser l'impact de certains des problèmes rencontrés sont présentées. Afin de présenter un portrait global, les avantages et les inconvénients de la réforme des cours tels que vécus par les membres du Département des sciences comptables de l'Université Laval sont présentés dans un tableau comparatif (Tableau 2).

\section{Les obstacles rencontrés}

L'implantation de la réforme ne s'est pas faite sans rencontrer d'embûches. En plus de la monopolisation des ressources humaines et matérielles visant à faciliter la transition entre les deux programmes, le Département des sciences comptables de l'Université Laval a fait face à plusieurs autres problèmes. Parmi ceux-ci, on retrouve la redéfinition de la tâche du professeur, la perception de son rôle, la perception de la réforme par les étudiants ainsi que par les autres départements de la Faculté des sciences de l'administration, l'évaluation d'habiletés et d'attitudes, et le manque de ressources financières et humaines qualifiées.

\section{La tâche du professeur et la perception de son rôle}

Traditionnellement, la tâche du professeur se composait d'activités de recherche et d'enseignement en plus de tâches administratives (voir Beaver, 1992). L'équilibre entre ces activités est important puisque toutes ces facettes de la carrière du professeur sont évaluées lors de l'octroi des promotions. Une conséquence directe de l'implantation de la réforme a été d'exiger une implication démesurée de certains professeurs dans le développement de matériel pédagogique. De plus, puisque le département avait déjà plusieurs postes à combler, plusieurs jeunes professeurs au début de leur carrière académique ont dû s'impliquer dans le développement de nouveaux cours. Ainsi, ces professeurs ont eu à 
Tableau 2

Tableau comparatif des avantages et inconvénients pour les professeurs impliqués dans la réforme des programmes au Département des sciences comptables de l'Université Laval

Avantages Inconvénients

- Défi intéressant à relever et d'une grande richesse intellectuelle tant cognitive qu'affective;

- Émergence, dans les premières années de l'implantation, d'une certaine effervescence auprès des professeurs s'impliquant activement dans la réforme;

- Augmentation des échanges, tant en quantité qu'en qualité, entre les professeurs donnant le même cours ou d'autres cours;

- Augmentation des échanges avec les praticiens et les étudiants;

- Les échanges favorisent

- une meilleure cohésion des contenus

- une vision globale du programme

- une augmentation de l'esprit d'équipe

- une entraide mutuelle et spontanée

- la cueillette d'une grande banque d'idées

- le développement d'attitudes, telles que l'empathie

intellectuelle, le respect du point de vue des autres et

l'ouverture d'esprit

- le développement d'habiletés de gestion de conflits;

- Mise à profit pour l'ensemble des membres de la créativité de chacun;

- Encourage la créativité pédagogique et la liberté académique;

- L'implantation (les trois premières années) monopolise pratiquement tous les effectifs du département à la création de nouveaux cours et du matériel didactique de soutien;

- Apparition progressive d'un certain essoufflement au fil des ans de l'implantation et du maintien de la réforme; diminution au même rythme de l'effervescence initiale;

- Augmentation importante de l'investissement en temps et énergie, par exemple:

- pour la création de nouveaux ateliers

- pour l'élaboration de nouveaux cas à chaque session

- pour les corrections formative et sommative plus élaborées et plus longues

- pour les rencontres pédagogiques entre collègues

- pour la disponibilité aux étudiants;

- Réunions très fréquentes pour permettre les échanges, la compréhension de chacun et les ajustements nécessaires concernant, entre autres, les objectifs et la planification des activités d'enseignement et d'apprentissage;

- La création de nouvelles activités ou du matériel didactique de soutien ainsi que les méthodes d'enseignement utilisées en classe doivent se faire en accord avec la philosophie, l'approche et les objectifs généraux de la réforme; 
- Favorise le développement d'une flexibilité dans la façon d'enseigner de chacun;

- Augmentation de la place accordée aux préoccupations pédagogiques dans l'esprit de chacun;

- Rapproche l'enseignement de la pratique sans délaisser la théorie comme fondement de base;

- Contribue à modifier l'image de transmetteur de connaissances à celle de guide dans la construction des connaissances de l'étudiant;

- Favorise la construction de matériel didactique inédit, intéressant et à jour au niveau de la théorie et de la pratique;

- Les échanges avec les étudiants permettent de mieux les connaître, de découvrir:

- leur calibre

- leurs intérêts

- leurs préoccupations

- leurs idées

- leurs connaissances initiales d'un sujet;

- Favorise chez les professeurs le développement d'habiletés et attitudes souhaitées chez l'étudiant; les habiletés et les attitudes démontrées par le professeur lors de résolutions de problèmes complexes et lors de discussion servent de modèle aux étudiants;
- Mal comprise, la réforme peut donner l'impression d'entraver la liberté académique;

- Manque de formation pédagogique ce qui rend plus difficile la compréhension et l'application du nouveau rôle du professeur en classe;

- Manque d'une compréhension profonde des finalités du nouveau programme;

- Manque de ressources humaines et financières;

- Augmentation de l'incertitude et de l'insécurité, car diminution du contrôle quant au déroulement du cours et aux contenus travaillés;

- Demande une flexibilité et une adaptabilité mises à l'épreuve à chaque séance de cours;

- Augmente la vulnérabilité du professeur due à l'ouverture aux points de vue et à la confrontation des idées avec les étudiants

- Demande une grande confiance en soi;

- Résistance au changement apparaissant d'une manière plus évidente pour certains;

- Résistance à mettre sur table ses façons de faire, à dévoiler ses «trucs»; 
- Les évaluations des étudiants sont considérées avec une plus grande attention; apparition d'un partage de l'information contenue dans les évaluations; utilisation de cette information combinée aux perceptions des professeurs quant au déroulement dans la classe pour évaluer les stratégies, les méthodes ou les activités qui fonctionnent bien ainsi que celles qui fonctionnent moins bien

- Les échanges entre collègues combinés au feedback oral des étudiants, à l'analyse des évaluations écrites et à la flexibilité qui caractérise l'idéologie de la réforme permettent un ajustement régulier des cours d'une session à l'autre et même d'une séance à l'autre.
- Demande des efforts et des encouragements constants - du renforcement de la part de la direction ou des pairs - pour ne pas retomber dans la sécurité de l'approche traditionnelle;

- Demande un encadrement plus serré des chargés de cours qui doivent, eux aussi, s'ajuster à la nouvelle philosophie d'enseignement;

- Le Département en tant qu'entité doit volontairement accepter une réduction temporaire des activités de recherche de ses membres au profit du développement de cours et de matériels didactiques de soutien;

- Possibilité pour les jeunes professeurs de ne pas rencontrer les critères de promotion en réduisant leur temps de recherche;

- Difficulté à recevoir les évaluations des étudiants critiquant personnellement un professeur qui pourrait être moins habile avec les nouvelles méthodes, stratégies ou activités d'enseignement. 
sacrifier partiellement leur recherche pendant une période de trois ans mettant incidemment en péril leurs chances de promotion.

Le rôle du professeur en classe ainsi que sa préparation ont changé. Un des objectifs de la réforme est de rattacher davantage les activités faites en classe à l'actualité. Par conséquent, les ateliers doivent être continuellement mis à jour ce qui exige donc un travail constant de révision et d'adaptation du matériel pédagogique. En fait, en adoptant du matériel pédagogique basé sur l'actualité financière, le professeur donne plus d'importance au développement d'outils pédagogiques et la coordination de cours devient ainsi plus exigeante et difficile à effectuer.

En adoptant une approche pédagogique favorisant la discussion et la communication en classe, le professeur est confronté à un niveau d'incertitude plus élevé que lors d'un cours magistral puisque le déroulement d'un cours ne peut plus être planifié en totalité. Le niveau d'incertitude est d'autant plus grand que le professeur doit intervenir en fonction des commentaires des étudiants. Étant donné que la majorité des professeurs n'ont pratiquement aucune formation en pédagogie, il est naturel qu'ils préfèrent opter pour une approche traditionnelle où ils ont le contrôle du contenu abordé et même du débit. ${ }^{11}$ Par conséquent, même si les professeurs se disent favorables à la nouvelle approche, l'introduction pragmatique de méthodes d'apprentissage différentes provoque de la part de certains professeurs une forte résistance au changement. D'ailleurs, même lorsque les professeurs adhèrent idéologiquement à la nouvelle approche, la tentation de retourner à l'ancienne méthode d'enseignement est toujours présente. En effet, le nouveau matériel didactique peut toujours être utilisé dans une approche plus traditionnelle; il n'est donc qu'un élément, nécessaire mais non suffisant, permettant de mettre en oeuvre une approche pédagogique. (On n'applique pas une approche, comme une recette, mais on la met en oeuvre et ceci, guidé par des principes)

Alors que certains problèmes rencontrés peuvent être minimisés en offrant aux enseignants des cours de pédagogie, certains obstacles demeureront. Un des problèmes les plus importants est la base de mesure utilisée lors des promotions pour évaluer la performance des professeurs. Malgré le discours officiel des universités qui soutiennent que la qualité de l'enseignement est un des facteurs importants dans l'établissement des 
critères de promotion, il s'agit d'un discours qui n'est pas mis en application puisque la décision d'agrégation repose principalement sur le dossier de recherche du professeur. On peut donc difficilement exiger qu'un jeune professeur réduise de façon marquée son implication dans la recherche et ainsi risquer qu'il soit pénalisé lors de l'agrégation. Un tel constat a été formulé par les membres du comité Bedford qui considèrent que les nouvelles approches pédagogiques préconisées ne peuvent pas être adoptées sans que les critères de promotion appuient de telles initiatives. Ces contradictions ont malheureusement pris figure de réalité au Département des sciences comptables de l'Université Laval.

La perception de la réforme par les étudiants et les autres départements

Méthodologie. Afin de recueillir la perception des étudiants face à la réforme des cours entreprise par le Département des sciences comptables de l'Université Laval, plusieurs groupes de discussions (focus groups) ont été formés. Dans la mesure du possible, chaque groupe de discussion était composé de huit à douze étudiants bénévoles et de deux à trois représentants du Département des sciences comptables. Afin de favoriser une discussion franche, le professeur responsable du cours discuté était absent. Dès le début de la séance, un représentant du Département des sciences comptables indiquait clairement aux étudiants que l'objectif recherché lors de ces discussions était l'amélioration du programme. De plus, il était mentionné que les commentaires spécifiques des étudiants ne seraient pas transmis à leur professeur et donc, qu'ils ne pourraient pas affecter leurs résultats scolaires.

Des groupes de discussion ont été formés pour les cours de première année et pour chacun des cours de deuxième et troisième années. Malgré toutes les précautions prises, il y a certaines limites associées à une telle démarche. Ainsi, puisque les étudiants se portaient bénévoles, un biais de sélection affecte probablement les résultats. De plus, malgré la garantie que leurs commentaires n'affecteraient pas leurs résultats scolaires, il se peut que les étudiants aient volontairement atténué leurs commentaires afin d'éviter d'être pénalisés dans leurs cours. Par contre, malgré ces limites, nous croyons que les commentaires recueillis lors de ces rencontres sont pertinents et représentatifs de la perception des étudiants. 
Résultats. Un des premiers commentaires négatifs notés lors des groupes de discussion est l'absence de technique comptable dans les cours de première année. Historiquement, les cours d'introduction à la comptabilité étaient consacrés à la tenue de livres et les étudiants associaient cette notion avec, ce qui était selon eux, la "vraie» comptabilité. Puisque les nouveaux cours mettent l'accent sur l'utilisation de la comptabilité et de ses produits dans la prise de décision au lieu de la préparation des états financiers, ils perçoivent les cours comme étant des cours sans orientation comptable précise. Le même phénomène s'est produit en fiscalité. Auparavant, le cours d'introduction à la fiscalité était bâti autour d'une série de règles fiscales. Le nouveau cours se penche davantage sur la structure de la loi et fait ressortir les liens entre ses différentes sections. Encore une fois, les étudiants ont mentionné, lors des groupes de discussion, que le nouveau cours ne pouvait pas les préparer à une carrière d'expert-comptable.

Ces problèmes de perception étaient amplifiés par les étudiants inscrits en parallèle dans l'ancien programme ou par ceux qui avaient déjà été exposés à la tenue de livres sur le marché du travail ou au collège. Les groupes de discussion traitant des cours plus avancés indiquent que cette mauvaise perception s'est en partie estompée puisque les étudiants devenaient visiblement de plus en plus conscients des objectifs d'apprentissage poursuivis par la réforme. En outre, puisque les cours plus avancés exposaient davantage les étudiants à l'aspect plus technique de la profession, ils ont également contribué à réduire leurs réticences. Nous croyons qu'une meilleure compréhension de l'utilité et de la pertinence de la réforme aurait tendance à modifier leur perception initiale de l'approche pédagogique utilisée et que plus d'efforts devraient donc être consacrés en ce sens.

D'après les commentaires notés lors des groupes de discussion, certains outils pédagogiques utilisés en classe ont également créé des problèmes de perception. Par exemple, certaines notions de comptabilisation des procédés de fabrication ont été abordées à l'aide de simulations. Une des activités demandait aux étudiants de fabriquer un produit à l'aide de matériaux de bricolage. Bien que cet atelier de manipulation permette aux étudiants de concrétiser certains concepts 
théoriques et de discuter de leur application, les étudiants percevaient cet atelier comme enfantin. De plus, cette perception a même été renforcée par des commentaires désobligeants de professeurs d'autres départements qui ne comprenaient probablement pas les objectifs visés par ces activités.

L'utilisation de problèmes complexes caractérisés, entre autres, par l'absence d'une solution unique mettait certains étudiants mal à l'aise. Puisque les étudiants étaient habitués à pouvoir identifier «la bonne réponse», le niveau d'incertitude associé aux problèmes complexes a créé une forte réaction négative, surtout de la part des étudiants n'ayant aucune expérience pratique. Cette réaction peut en partie s'expliquer par un développement post-formel inachevé. En effet, selon un modèle cognitif du développement en sept stades (King \& Kitchener, 1994), les étudiants réagissant ainsi seraient au stade deux ou trois, c'est-à-dire qu'ils auraient une vision dichotomique de la réalité: il y a les solutions vraies, les solutions fausses. À la limite, ils admettent que la véracité d'une solution soit encore incertaine, mais ce n'est qu'une question de temps avant qu'on puisse déterminer son statut. De plus, ces étudiants s'en remettent facilement aux experts qui eux détiendraient la vérité. À l'opposé, les étudiants ayant atteint le stade 6 ou 7 sont capables de mettre en perspective le contexte, les intervenants, les éléments factuels, les valeurs impliquées et de prendre en considération les incertitudes ou le manque d'information, les aspects éthiques, économiques, politiques et légaux d'une situation. Ils ne sont pas à la recherche de «la» bonne solution, mais de la solution jugée comme acceptable par les pairs, compte tenu du contexte. Ils n'essaient pas non plus d'appliquer des recettes mais plutôt des stratégies générales (heuristiques) de résolution de problèmes.

Les problèmes de perception ont été amplifiés par le fait que la réforme du cheminement comptable s'inscrivait dans un programme de baccalauréat en administration des affaires. Ainsi, les étudiants en sciences comptables ne prenaient que 13 cours en comptabilité sur un total de 32 cours au baccalauréat. Puisque les autres départements de la Faculté des sciences de l'administration ont presque tous conservés une approche traditionnelle, les étudiants étaient confrontés à deux approches pédagogiques différentes. Les cours du nouveau programme mettent l'emphase sur la discussion et, dans ces circonstances, l'étudiant 
démontre certaines difficultés à synthétiser l'information et à conceptualiser les diverses notions. De façon générale, les étudiants ont apprécié le dynamisme des nouveaux cours. Toutefois, étant donné que certains étudiants évaluent leur niveau de connaissance dans un cours par la quantité de notes prises, ils avaient l'impression de ne pas apprendre autant dans les cours de comptabilité que dans les autres cours. De plus, l'absence de notes abondantes dans certains cours de comptabilité augmentait leur niveau d'incertitude face aux examens, créant ainsi un certain climat de méfiance et d'insatisfaction. Il est aussi possible que le style cognitif des étudiants attirés par la comptabilité soit davantage celui de convergeur (exécution minutieuse de tâches avec protocole, approche linéaire, applications concrètes, travail en solitaire) que celui de divergeur (analyse de faits selon diverses perspectives, imagination et créativité, intérêt envers les personnes, etc.) (Kolb, 1985).

Finalement, d'après les commentaires notés lors des groupes de discussion, la proportion élevée de temps consacré au travail en équipe a également créé un niveau d'insatisfaction dû, entre autres, au manque de sérieux de certains étudiants et de l'incompréhension des objectifs recherchés par cette approche. Un des problèmes soulevés était l'écart important existant entre le niveau de préparation des étudiants lors des discussions en équipe. Alors que certains étudiants se préparaient adéquatement avant de se présenter en classe, d'autres profitaient du temps alloué pour la discussion en équipe pour lire la donnée du problème. Dans certains cas extrêmes, les étudiants ne discutaient simplement pas de l'activité. De plus, ne comprenant pas les objectifs pédagogiques à atteindre par ces activités, certains étudiants considéraient ces discussions comme étant inutiles.

\section{L'évaluation d'habiletés et d'attitudes}

Malgré tous les changements importants apportés au cheminement des sciences comptables pour atteindre le profil du professionnel décrit dans le rapport Bedford et la première prise de position de l'AECC, il reste encore certains objectifs pédagogiques qui ne sont pas atteints et évalués. Dans un premier temps, le nouveau programme n'intègre pas d'activités ciblant directement les processus de pensée. Les étudiants sont effectivement soumis à des activités de réflexion et de résolution de 
problèmes favorisant le développement de la pensée. Cependant, aucune activité n'enseigne la pensée en elle-même ou la manière de la gérer. Par exemple, même si l'étudiant est appelé à évaluer de l'information et sa source, cette tâche n'est pas explicitement décrite en classe comme étant intégrée au contenu à apprendre et elle n'est pas évaluée. De la même manière, les différentes activités d'apprentissage ne mettent pas en relief la façon de penser des étudiants et ne les amènent pas à faire de l'autoévaluation et de l'autorégulation de leur propre processus de pensée. ${ }^{12} \mathrm{En}$ outre, bien que certaines attitudes liées au professionnalisme, telle la rigueur intellectuelle, sont fort probablement acquises par l'étudiant exposé aux différentes activités, le contenu des cours et du programme présentent très peu d'objectifs spécifiques à cet égard.

Dans un deuxième temps, diverses difficultés au niveau de l'évaluation ont été rencontrées. Malgré la diversité des méthodes d'évaluation, nous déplorons le manque d'évaluation formative. Bien que fort utile et même essentielle à la formation de l'étudiant, elle exigeait trop de temps et d'énergie des professeurs. Donc, même si l'ajout d'évaluation formative représentait une amélioration importante par rapport à l'ancien programme, il aurait lieu d'y consacrer plus d'efforts. Cette situation est en partie causée par le fait que les travaux demandés, presque tous des productions complexes, sont probablement trop longs à commenter. ${ }^{13}$

En général, les étudiants ont apprécié la diversité des méthodes d'évaluation. Toutefois, leur perception quant au degré d'objectivité de ces méthodes a créé certains problèmes. Par exemple, même si on voulait rendre l'étudiant plus actif en classe et l'inciter à verbaliser sa pensée, sa participation a fait l'objet d'une évaluation sommative qui représentait généralement $10 \%$ de la note globale. Cette évaluation soulignait certaines attitudes comme le respect de l'opinion des autres, et des habiletés de communication orale. En plus des difficultés propres à ce type d'évaluation, telle la détermination de critères objectifs, les professeurs ont été fortement critiqués par les étudiants qui percevaient celle-ci comme subjective, peu fondée et souvent injuste. En fait, la perception des étudiants entendue lors des groupes de discussion était qu'ils avaient droit à ces points quel que soit leur comportement en classe. ${ }^{14}$ En outre, la taille des groupes pouvant atteindre près de 60 étudiants dans certains cas rendait cette tâche d'évaluation beaucoup plus difficile. 


\section{Le manque de ressources financières et humaines qualifiées.}

La plupart des universités américaines qui ont réformé en totalité les programmes en sciences comptables ont bénéficié de ressources monétaires importantes de la part des organismes comptables. ̇̀ l'opposé, peu de ressources étaient disponibles pour entreprendre une telle réforme à l'Université Laval. Par exemple, l'adaptation et la traduction du matériel pédagogique utilisé dans les cours de première année a coûté près de $80000 \$$ au Département des sciences comptables. Pour lui permettre de mener à terme cette tâche, le Département a reçu $5000 \$$ de subvention de l'Ordre des comptables agréés du Québec et $30000 \$$ de fonds interne. ${ }^{15}$ Pour le développement des cours des autres années, pratiquement aucune aide n'a été octroyée au Département. Considérant qu'au total 12 cours ont été réformés, la grande majorité des coûts associés au développement de matériel didactique a été absorbée par le Département sous la forme de surcharge de travail de la part des professeurs. De plus, malgré l'engagement de deux comptables expertes en pédagogie, les ressources humaines qualifiées restaient toujours insuffisantes, ce qui n'a permis aux membres du Département de déléguer suffisamment les tâches de conception de matériel didactique. Ce facteur, combiné au fait que plusieurs postes de professeurs dans le département des sciences comptables n'étaient pas comblés, a résulté en un essoufflement généralisé des professeurs actifs.

\section{Les réussites et les améliorations possibles}

Malgré les difficultés rencontrées lors de la réforme de la formation comptable, nous croyons fermement que les modifications apportées au programme aideront les étudiants tout au long de leur carrière. La réforme a d'autant plus de mérite qu'elle a été instaurée avec peu de moyens financiers et humains. Des avantages inattendus se sont présentés. Par exemple, le fait de travailler conjointement à un programme a permis une plus grande cohésion et complicité entre les membres du Département. En fait, la réforme a suscité chez le corps professoral une certaine effervescence, surtout au début de sa mise en place. Elle a donné lieu a de nombreux échanges entre les professeurs ce qui a même résulté dans l'élaboration de projets de recherche conjoints. 
L'ajout de deux expertes en pédagogie dans le Département a permis une plus grande compréhension des nouvelles approches pédagogiques chez les professeurs. De plus, la refonte des cours a développé une plus grande conscience de la pédagogie dans les préoccupations quotidiennes des membres du Département. L'importance accrue accordée à l'enseignement a été d'ailleurs très appréciée des étudiants. En fait, les nombreux échanges en classe ont créé une plus grande complicité entre le professeur et les étudiants. Ces derniers pouvaient s'identifier plus facilement à leurs professeurs. Les étudiants ont également apprécié le dynamisme des nouveaux cours puisqu'ils étaient appelés à y jouer un rôle actif. Certains d'entre eux ont également apprécié les discussion en équipe puisqu'elles permettaient d'échanger leurs idées tout en améliorant leur compréhension de la matière. En outre, certaines activités pédagogiques «plus osées» ont relativement bien fonctionné et ont été appréciées par les étudiants. Ce fut la cas, par exemple, des jeux de rôle et des débats.

Malgré l'importance des avantages associés à une telle réforme, nous croyons que les conséquences fầcheuses, dues entres autres aux nombreux obstacles rencontrés, doivent être réduites afin d'assurer le succès d'une telle réforme. L'expérience que nous avons vécue nous incite à croire que certaines stratégies peuvent être mises en place afin de faciliter l'implantation de tels changements. Une grande partie des problèmes rencontrés lors de l'implantation de la réforme est probablement due au manque de promotion de celle-ci. De plus grands efforts auraient été nécessaires afin de publiciser les intentions, la philosophie, les objectifs de la réforme ainsi que ses accomplissements tant à l'externe (ex.: autres universités, Ordres professionnels comptables, bureaux comptables) qu'à l'interne par la voie de présentations lors de congrès, de communiqués ou articles dans des journaux scientifiques, professionnels, facultaires et universitaires. Une telle promotion aurait pu générer un support financier du milieu des affaires ainsi qu'une certaine forme de partenariat. En outre, une meilleure connaissance de nos efforts à l'externe aurait pu favoriser une affiliation avec d'autres universités pour le développement de matériel pédagogique. À l'interne, une meilleure compréhension de la réforme aurait certainement amoindri certains problèmes de perception tout en favorisant l'harmonisation entre les cours offerts par le Département des 
sciences comptables et ceux offerts par les autres départements de la Faculté des sciences de l'administration.

Le manque de formation en pédagogie des enseignants a également rendu la tâche plus difficile. Ainsi, en étant conscients des stades de développement cognitif des étudiants, on choisirait des activités pédagogiques davantage ciblées en fonction de leurs besoins. Il serait également plus aisé de faire comprendre aux étudiants les objectifs poursuivis par la réforme des cours. Afin de combler cette lacune, il serait souhaitable de développer des projets avec la Faculté des sciences de l'éducation. De plus, certains problèmes auraient pu être éliminés en créant davantage de collaboration entre les universités, américaines ou canadiennes, qui ont procédé à de telles réformes. Il aurait été alors possible d'éviter certains pièges déjà identifiés par d'autres universités.

Il est présentement difficile de bien évaluer les changements apportés au cheminement des sciences comptables. Pour l'instant, la source la plus importante d'information est celle générée par les groupes de discussion. Tel que mentionné précédemment, cette source d'information est probablement partiellement biaisée. Un autre indicateur du succès du nouveau programme est le taux de réussite des étudiants aux examens des ordres comptables. Par contre, on doit être très prudent avec cette statistique puisque l'objectif premier du changement apporté au programme était de mieux préparer l'étudiant à devenir professionnel et non pas à mieux performer lors de ces examens. Malgré tout, le taux de réussite des étudiants aux examens de 1997, soit la première année ou des finissants du nouveau programme se présentaient aux examens, était semblable à celui des années antérieures. En fait, l'évaluation la plus pertinente du nouveau programme devra venir des employeurs. Ainsi, dans quelques années, il sera possible d'avoir une meilleure mesure de l'atteinte de nos finalités en mesurant le degré de satisfaction des employeurs face aux étudiants diplômés des sciences comptables de l'Université Laval. Malheureusement, il est encore trop tôt pour pouvoir utiliser cette source d'information. 


\section{CONCLUSION}

L'objectif de cet article est de présenter l'expérience vécue par le Département des sciences comptables de l'Université Laval lors de la réforme de son programme. Plusieurs facteurs ont incité le Département des sciences comptables à modifier en profondeur les cours offerts. Parmi ces facteurs, on retrouve l'écart grandissant entre les besoins des employeurs et la formation des étudiants, les changements vécus par la profession comptable et les faiblesses des étudiants face à l'intégration de la matière, à la compréhension du cadre théorique et au jugement critique. Ces facteurs, combinés à la réflexion américaine sur l'enseignement de la comptabilité, ont contribué au développement d'un nouveau programme.

La structure du nouveau programme est conforme aux recommandations de divers comités américains qui se sont penchés sur la formation en sciences comptables. Plus précisément, la formation au cours de la première année est générale et elle devient plus spécialisée à la fin du programme. En plus d'avoir modifié le contenu des cours, l'approche pédagogique a également changé. Alors que l'ancien programme avait une approche traditionnelle où l'étudiant était surtout passif, le nouveau programme met l'accent sur l'autonomie des étudiants en favorisant l'émergence du jugement professionnel ainsi que les habiletés et les attitudes qui y sont rattachées. Le programme est axé sur une compréhension globale favorisant l'intégration des connaissances.

Plusieurs obstacles ont été rencontrés lors de l'implantation du programme. Ainsi, les professeurs du Département des sciences comptables ont dû consacrer une partie anormalement élevée de leur temps au développement de matériel pédagogique et ceci, au détriment de leurs autres activités académiques. De plus, en adoptant une approche pédagogique basée sur la participation et la communication, le professeur fait face à un niveau d'incertitude en classe qui est plus important que lors de l'enseignement d'un cours magistral. La réticence face aux changements de la part de certains professeurs a aussi été un obstacle important lors de la réforme.

La perception des étudiants face à la réforme a également été un problème. Par exemple, l'absence de technique comptable dans les cours de première année a apporté beaucoup de confusion chez les étudiants 
car cela allait à l'encontre de leurs attentes. L'utilisation d'ateliers demandant aux étudiants de fabriquer un produit à l'aide de matériaux de bricolage afin de concrétiser certaines notions abstraites a aussi été perçue comme enfantin tant par les étudiants que par des professeurs d'autres départements de notre faculté. En fait, les objectifs pédagogiques recherchés par ces activités n'ont pas été compris. Également, la présence de problèmes complexes caractérisés par l'absence de solution unique mettait les étudiants mal à l'aise. La réaction des étudiants et des professeurs d'autres départements de la Faculté des sciences de l'administration suggère qu'un élément crucial du succès d'une telle réforme réside en la capacité de bien faire comprendre les objectifs recherchés et d'en faire la promotion.

Un autre problème majeur que nous avons rencontré lors de l'implantation de la réforme a été le manque de ressources. Notre expérience nous a fait comprendre l'importance d'avoir non seulement des ressources monétaires et humaines suffisantes, mais la nécessité d'avoir l'appui inconditionnel des instances supérieures. Notre réforme a été effectuée dans un contexte où tous ces éléments n'étaient pas réunis. La conséquence directe fut de créer une surcharge de travail de la part des professeurs résultant en un essoufflement précoce. Au début de ce projet d'envergure, nous avons remarqué une certaine effervescence qui, à défaut d'un support approprié et soutenu, a entraîné un certain relâchement incitant même certains professeurs à retourner à une approche traditionnelle. L'appui des instances supérieures de l'Université est également crucial puisque les critères de promotion doivent nécessairement récompenser ce genre d'initiative si on désire avoir un engagement de la part des jeunes professeurs.

Malgré tous les obstacles rencontrés, nous croyons fermement que le nouveau programme aidera les étudiants à être plus autonomes face à leur apprentissage et à devenir de meilleurs professionnels. En écrivant cet article, nous voulons non seulement partager notre vécu mais nous désirons également exposer les difficultés auxquelles peut s'attendre tout département qui décide d'innover en pédagogie et du même coup proposer certains éléments de solution. 


\section{Notes}

1 L'American Accounting Association est un organisme comptable qui regroupe principalement des professeurs de comptabilité et certains praticiens. En plus d'organiser plusieurs congrès axés sur la recherche en sciences comptables, cet organisme publie différentes revues scientifiques.

2 L'absence de réforme des programmes en comptabilité au Canada s'explique en partie par la rareté du financement disponible, contrairement aux États-Unis, pour de telles refontes en sciences de l'administration.

3 Les problèmes complexes ou non structurés sont ceux où il existe une pluralité indéterminée de solutions possibles sans que l'on puisse jamais savoir si l'on a trouvé la meilleure. Ils impliquent qu'il y a peu ou pas d'information sur la situation initiale, la situation finale ou les opérateurs (Legendre, 1993).

4 L'University of Southern California a reçu une subvention de $250000 \$$ de la Coopers \& Lybrand Foundation pour la réforme de leur programme en sciences comptables.

5. Bien qu'incluse dans la liste des attitudes par l'AECC, la créativité s'apparenterait mieux à la notion de style cognitif, de mode de pensée ou d'une compétence à développer.

6 En fait, une traduction plus juste du terme utilisé dans les publications comptables sur la formation serait probablement «apprentissage à apprendre» (learning to learn). Cette notion apparaît dans la plupart des documents qui sont à l'origine des changements dans la formation en sciences comptables. Cependant, il s'agit d'un concept complexe qui n'était pas expliqué dans ces documents. Dans cette optique, l'AECC et l'AAA ont publié, en 1995, un document explicite à ce sujet. Le groupe de travail à l'origine de ce texte utilise l'appellation «apprentissage intentionnel» (intentional learning). Il décrit la nature du processus, son utilité dans la formation universitaire et les conditions favorisant son développement chez l'étudiant. Les auteurs font aussi référence à son homologue dans le domaine de la psychologie cognitive, soit le concept de métacognition. Ces auteurs définissent l'apprentissage intentionnel comme un processus d'acquisition, de compréhension et d'utilisation de stratégies variées dans le but d'améliorer sa propre habileté à acquérir et à appliquer des connaissances. Ce processus résulte de, conduit à et développe l'esprit de questionnement ainsi qu'un désir d'apprendre toute la vie durant. Un étudiant qui pratique l'apprentissage intentionnel, c'est-à-dire qui gère son propre processus d'apprentissage, démontre une intention de vouloir apprendre, et choisit ce qu'il apprendra, comment ainsi que quand il le fera. 
7 Traditionnellement, les universités francophones du Québec ont fait bande à part en matière d'enseignement de la comptabilité. En effet, le cheminement que doivent suivre les étudiants des universités francophones du Québec désirant obtenir le titre de comptable agréé n'est pas le même que celui imposé aux étudiants des universités anglophones. À titre d'exemple, les universités francophones du Québec permettent à leurs étudiants de se présenter aux examens des corporations professionnelles immédiatement après l'obtention du baccalauréat alors que les étudiants des autres universités doivent d'abord compléter leur stage de formation pratique, d'une durée de deux ou trois ans dépendant des provinces.

8 Ces laboratoires étaient davantage des séances de résolution de problèmes au tableau par un assistant de cours et les étudiants avaient très souvent un rôle passif.

9 Plusieurs universités américaines se sont contentées de réformer les cours de première année tout en introduisant des cours d'appoints entre la première et la deuxième années afin d'insérer les anciens cours de deuxième année. La plupart des universités ayant réformer en totalité leur programme de premier cycle sont celles qui ont été financées par l'AECC.

10 Afin de favoriser les discussions en équipe dans la salle de cours, deux locaux ont été équipés de tables rondes. De plus, l'ajout de matériel informatique et audiovisuel a été nécessaire.

11 La plupart des programmes de doctorat en comptabilité en Amérique de Nord se limitent à l'enseignement des outils de recherche et pratiquement aucun cours de pédagogie n'y est dispensé.

12 Selon Costa (1985), il est nécessaire de combiner trois grands types d'enseignement pour favoriser le développement de la pensée: (a) l'enseignement pour penser (teaching for thinking): le professeur crée en classe les conditions favorables à la pensée des étudiants, par exemple en faisant usage de techniques d'enseignement stimulantes pour la pensée, telles que la discussion, la résolution de problèmes, l'expérimentation et l'écriture; (b) l'enseignement de la pensée (teaching of thinking): le professeur enseigne explicitement les habiletés intellectuelles ou les processus de pensée visés en élaborant, par exemple, un modèle descriptif des opérations de pensée; (c) et l'enseignement à propos de la pensée (teaching about thinking): le professeur aide les étudiants à devenir plus conscients de leurs propres processus cognitifs et de ceux des autres, de leur évaluation et de leur régulation, de même que l'utilisation de ces processus dans les problèmes et les situations de la vie réelle. 
Actuellement, la réforme implantée en sciences comptables n'a bien exploitée que le premier de ces types d'enseignement.

13 Le temps nécessaire au développement de matériel pédagogique et l'évaluation de l'acquisition des compétences chez l'étudiant explique en partie le peu de réformes en profondeur des programmes en sciences comptables dans les universités canadiennes.

14 La perception négative des étudiants à l'égard de l'évaluation de la participation était amplifiée par le fait que les cours offerts par les autres départements n'évaluaient généralement pas cette dimension de l'apprentissage.

15 En plus, un montant de $50000 \$$ a été offert par le Fonds d'investissement étudiant de la Faculté des sciences de l'administration pour les changements d'ordre physique de l'environnement pédagogique dans les salles de cours notamment, des tables rondes ainsi que du matériel informatique et audiovisuel.

\section{Références}

Accounting Education Change Commission (AECC). (1990). Issues Statement No. 1: AECC Urges Priority for Teaching in Higher Education.

Accounting Education Change Commission (AECC) and American Accounting Association (AAA). (1995). In M. C. Francis, T.C. Mulder, \& J.S. Stark (Eds.), Intentional learning A process for learning to learn in the accounting curriculum. Accounting Education Series, Vol. 12.

American Accounting Association, Committee on the Future Structure, Content, and Scope of Accounting Education (the Bedford Committee). (1986). Future Accounting Education: Preparing for the Expanding Profession. Issues in Accounting Education, I(1), 168-90.

Anderson T., \& Taylor, L. (1994). Enseigner la résolution de problèmes. CA Magazine, 127(5), 39-46.

Arthur Anderson \& Co., Arthur Young, Coopers \& Lybrand, Deloitte Haskins \& Sells, Ersnt \& Whinney, Peat Marwick Mitchell \& Co., Prince Waterhouse, and Touche Ross (1989). Perspectives on education: Capabilities for success in the accounting profession.

Beaver, W.H. (1992). Challenges in accounting education. Issues in Accounting Education, 7, 135-143.

Costa, A.L. (1985). Teaching for, of, and about thinking. In A.L. Costa (Ed.), Developing minds. A resource book for teaching thinking, (pp. 20-23). Alexandria, VA: Association for Supervision and Curriculum Development. 
Friedlan, J. (1995). Fausse représentation. CA Magazine, 128(7), 48-52.

Guilbert, L., \& Ouellet, L. (1997). Étude de cas - Apprentissage par problèmes. Québec, QC: P.U.Q.

Institut canadien des comptables agréés du Canada, Groupe de travail pancanadien sur la vision de la profession. (1996). Rapport de recherche.

International Federation of Accountants. (1995). Integrating information technology across the accounting curriculum: The experience of the Certified General Accountants' Association of Canada.

King, P.M., \& Kitchener, K.S. (1994). Developing reflective judgment. San Francisco, CA: Jossey-Bass.

Kolb, D.R. (1985). The learning style inventory. Boston, MA: McBer and Co.

Legendre, R. (1993). Dictionnaire actuel de l'éducation. Montréal, QC: Guérin.

Patten, J.R., \& Williams, D.Z. (1990). There's trouble right here in our accounting programs: The challenge to accounting educators. Issues in Accounting Education, 5, 175-179.

Schön, D.A. (1987). Educating the reflective practitionner: Toward a new design for teaching and learning in the professions. San Francisco, CA: Jossey-Bass.

Sundem, G.L. (1994). Repenser la formation en comptabilité, $C A$ Magazine, 127(3), 41-46.

Williams, D.Z. (1993). Reforming accounting education. Journal of Accountancy, 176(2), 76-82.

\section{Annexe}

\section{Recommandations du rapport Bedford}

L'analyse du comité au sujet des pratiques comptables a indiqué que l'approche de formation en sciences comptables requiert des ajustements majeurs d'ici l'an 2000. Les recommandations contenues dans ce rapport sont destinées à servir d'indicateurs et à fournir des directives pour ceux qui ont pris l'initiative d'apporter des changements à la formation en sciences comptables. Étendre les responsabilités à tous les intervenants de la profession fournit en retour un élargissement des possibilités pour les formateurs en sciences comptables. 


\section{Étendue et contenu}

1. La comptabilité devrait être vue comme un vaste processus de développement et de communication d'information économique, basée sur la conception, l'implantation et l'exploitation de différents types de systèmes d'information. De ce fait, les professeurs de comptabilité devraient s'assurer de conserver des compétences dans le domaine des technologies de l'information et maintenir leurs efforts dans le développement de systèmes complets d'information pour les organisations.

2. Les membres du corps professoral en comptabilité devraient reconnaître et aviser les étudiants qu'une formation rigoureuse en matière de comptabilité générale et de développement de compétences individuelles élargies sont préférables à une spécialisation prématurée en sciences comptables.

3. Les membres du corps professoral en comptabilité devraient être réceptifs à l'élargissement des exigences d'enseignement des sciences humaines et des sciences pures, et ce en vue de développer chez l'étudiant des compétences à l'analyse, à la synthèse, à la résolution de problèmes et à la communication.

4. La formation universitaire en sciences comptables devrait mettre l'emphase sur les habiletés et les compétences nécessaires à un processus d'apprentissage permanent.

5. Les objectifs pédagogiques des programmes et des cours devraient être construits de manière à aider les étudiants à apprendre à apprendre, à développer leur pensée et à être créatifs.

6. Les membres du corps professoral en comptabilité devraient établir des exigences élevées pour les étudiants et ajuster les programmes et les méthodes d'apprentissage en vue de développer les compétences professionnelles et personnelles ainsi que les connaissances générales souhaitées chez les étudiants.

7. Les universités devraient maintenir un enseignement des sciences comptables flexible afin de s'ajuster rapidement aux besoins d'information constamment en changement dans notre société.

\section{Structure}

8. La structure des programmes devrait être suffisamment large pour englober trois degrés de formation:

- une formation en sciences humaines, en arts et en sciences pures, soit une formation générale; 
- une formation générale permettant le développement des concepts concernant la recherche, l'organisation et la communication d'informations comptables, soit la formation professionnelle générale requise de tout professionnel comptable;

- et une formation visant des connaissances techniques spécialisées dans une ou plusieurs des disciplines de la comptabilité, soit la formation professionnelle comptable spécialisée.

9. La formation professionnelle comptable spécialisée devrait être offerte exclusivement à un niveau supérieur d'enseignement. Dès lors, l'intégration complète des trois degrés de formation dans un programme de comptabilité devrait normalement s'échelonner sur un minimum de cinq ans.

10. Les praticiens et les professeurs-chercheurs en comptabilité devraient se baser sur le principe de l'avantage comparatif pour choisir le contenu spécialisé qui sera dispensé par les universités et celui qui sera dispensé par les employeurs ou à travers des programmes de formation continue.

\section{Aspects pédagogiques, administratifs et professionnels}

11. Les activités d'apprentissage devraient requérir des étudiants qu'ils soient actifs et autonomes dans leur apprentissage et qu'ils puissent faire de la résolution de problèmes plutôt que d'être des récipients passifs d'information.

12. La formation universitaire en sciences comptables devrait concevoir des activités d'apprentissage et attribuer un certain degré de responsabilité aux professeurs en vue d'augmenter l'interaction entre étudiants et professeurs sur des enjeux intellectuels, favorisant ainsi le développement personnel des étudiants.

13. Le matériel didactique comptable, telles les notes de cours, devrait être construit pour refléter la structure future, le contenu et l'étendue des programmes de formation en sciences comptables.

14. Chaque programme universitaire en comptabilité devrait mettre des priorités appropriées sur l'évaluation des apprentissages étudiants, la recherche et le service aux ordres professionnels, et ce en accord avec les finalités de l'université et les changements requis par une profession en mutation.

15. La formation en sciences comptables devrait supporter la recherche utile au développement des connaissances dans la profession. En ce sens, la recherche pure et la recherche appliquée devraient être encouragées et supportées. 
16. Les universités devraient établir des standards élevés pour l'admission aux programmes en sciences comptables. Ceux-ci pourraient même inclure des critères indépendants de ceux des autres programmes.

17. Les universités devraient attribuer plus de ressources (ex.: professeurs de carrière et taille restreinte des groupes ) pour les cours d'introduction à la comptabilité et des cours intermédiaires; redéfinir et élargir le rôle des professeurs dans la structure éducative en vue d'augmenter leur implication dans l'apprentissage des étudiants; offrir à ces derniers un encadrement et des conseils systématiques dès l'inscription jusqu'à la diplômation.

18. Les universités devraient attribuer des ressources à des programmes et des activités complémentaires qui permettent de motiver les étudiants et qui augmentent la diversité des expériences d'apprentissage.

19. Les universités devraient implanter l'évaluation par les pairs des programmes en sciences comptables, soit par des professeurs provenant d'autres disciplines ou des professeurs en sciences comptables d'autres institutions et par des praticiens.

20. Les universités devraient encourager une plus grande interaction des professeurs en sciences comptables entre eux et avec ceux des sciences sociales.

21. Les universités devraient encourager le développement de programmes de cycles supérieurs en sciences comptables.

22. Les universités devraient mettre l'emphase sur la valorisation et l'utilisation par les professeurs de ressources éducatives d'appoint en provenance des sciences humaines et des sciences pures.

23. Les universités devraient permettre la décentralisation des programmes et fournir plus d'autonomie aux enseignants en sciences comptables en ce qui a trait, par exemple, à l'établissement des critères d'admission et de rétention supérieurs à ceux de l'université afin que les programmes en sciences comptables puissent s'adapter plus rapidement aux changements de l'éducation professionnelle.

24. Réduire la technique comptable aux seuls cours ou domaines requis pour l'accréditation.

25. En préparant les étudiants à leur future vie professionnelle, les facultés en sciences comptables devraient se baser sur la pratique, la recherche en sciences comptables et la recherche multidisciplinaire pour construire le 
contenu des programmes plutôt qu'uniquement sur la préparation immédiate des étudiants à l'exercice de la pratique actuelle.

26. Les employeurs des futurs experts-comptables devraient avoir une meilleure compréhension de l'environnement économique propre à la formation en sciences comptables ainsi que des coûts et des bénéfices découlant des changements de politique des universités concernant les programmes de formation en sciences comptables, non seulement au premier cycle mais aussi aux deuxième et troisième cycles.

27. Les institutions devraient considérer des structures qui encouragent une recherche diversifiée et vitale de la part des professeurs-chercheurs, tout en respectant la liberté académique et les règles d'agrégation.

28. Des subventions de recherche devraient être accordées à des projets qui tentent d'expliquer raisonnablement l'environnement économique propre à la formation en sciences comptables. 